\title{
Annealed Co thin films: Pit formation and magnetic anisotropy
}

H. Shi

D. Lederman

Follow this and additional works at: https://researchrepository.wvu.edu/faculty_publications

\section{Digital Commons Citation}

Shi, H. and Lederman, D., "Annealed Co thin films: Pit formation and magnetic anisotropy" (2000). Faculty Scholarship. 57. https://researchrepository.wvu.edu/faculty_publications / 57 


\title{
Annealed Co thin films: Pit formation and magnetic anisotropy
}

\author{
H. Shi and D. Lederman \\ Department of Physics, West Virginia University, Morgantown, West Virginia 26506-6315
}

We present a study of the morphological and magnetic transformation of hcp(0001) Co thin films, grown via sputtering on $\mathrm{Al}_{2} \mathrm{O}_{3}(11 \overline{2} 0)$, which occurs when they are annealed in vacuum in the temperature range of $500{ }^{\circ} \mathrm{C}<T_{A}<600{ }^{\circ} \mathrm{C}$. In this temperature range, the films undergo a reentrant smoothening transition. For very thin films (thickness $t<20 \mathrm{~nm}$ ), this coincides with a fcc(111) to fcc(001) recrystallization, and with the appearance of rectangular pits. For thicker films, the recrystallization does not occur, even though the surface smoothens out leaving hexagonal pits behind. The number of pits per unit area decreases exponentially as the thickness of the film is increased, demonstrating that interactions at the film surface and film-substrate interface cause the smoothening and pit formation during the annealing process. Whereas the $\mathrm{hcp}(0001)$ and fcc(111) phases are magnetically isotropic in the film's plane, the fcc(001) films exhibit a biaxial in-plane anisotropy. (C) 2000 American Institute of Physics. [S0021-8979(00)50808-1]

\section{INTRODUCTION}

Co thin films have been intensively studied during the past few years because of the ease of epitaxial growth on different substrates, its applications in giant magnetoresistive (GMR) devices, and in the formation of magnetic nanoparticles. Bulk Co is unusual among the $3 d$ transition metal elements because it undergoes a hcp to fcc martensitic transition at a temperature $\approx 415^{\circ} \mathrm{C}$.

Much of the research on pure Co films has focused on the crystallinity and film quality of as-grown samples. It has been previously reported that $\mathrm{Co}$ films deposited on $\mathrm{Al}_{2} \mathrm{O}_{3}(11 \overline{2} 0)$ at approximately $300^{\circ} \mathrm{C}$ are smoother than films grown at higher temperatures, ${ }^{2}$ and that the fcc phase is stable in fine particles only at high temperatures. ${ }^{3}$ It is also known that the fcc Co phase can be stabilized at low temperatures in $\mathrm{Co} / \mathrm{Ni}$ superlattices, ${ }^{4}$ and that bcc Co can be grown on GaAs as single films ${ }^{5}$ or in $\mathrm{Co} / \mathrm{Fe}$ superlattices. ${ }^{6}$

We recently discovered a new reentrant smoothening transition in pure Co films grown on $\mathrm{Al}_{2} \mathrm{O}_{3}(11 \overline{2} 0) .{ }^{7}$ In this case, as-grown epitaxial films annealed at higher temperatures initially become rough as the annealing temperature $T_{A}$ is increased. However, in the temperature range $500{ }^{\circ} \mathrm{C}$ $<T_{A}<600^{\circ} \mathrm{C}$, the surface smoothens out, leaving submicron-sized rectangular pits behind if the film thickness $t<20 \mathrm{~nm}$. This coincides with a fcc(111) to fcc(001) recrystallization of the lattice. A similar, thus far unexplained smoothening transition, which leaves nanometer-scale rectangular pits behind, occurs in $\mathrm{Ag}$ fcc(111) films grown at $130 \mathrm{~K}$ on GaAs when they are annealed at room temperature. $^{8}$

By understanding the basic mechanism responsible for the pit formation in these materials, new lithographic techniques, and novel magnetic structures could be developed. In this paper, we study further the morphology and magnetic properties of annealed Co films to obtain further clues about the pit formation mechanism. We find that the concentration of rectangular pits in fcc(001) films decreases exponentially with the film thickness, indicating that the pits form because of a surface or surface/substrate interaction. We also find that thicker films do not undergo the fcc(111) to fcc(001) reorientation, although the smoothening transition does occur and hexagonal pits are formed. The fcc(001) recrystallized films are magnetically anisotropic in the plane, in contrast with the isotropic fcc(111) and hcp(0001) films.

\section{EXPERIMENTAL DETAILS}

The samples were grown via dc magnetron sputtering in a high vacuum chamber with base pressure of 3 $\times 10^{-7}$ Torr using a $3.0 \mathrm{mTorr}$ argon pressure. The growth temperature for all samples was $315^{\circ} \mathrm{C}$, and the substrate was $\mathrm{Al}_{2} \mathrm{O}_{3}(11 \overline{2} 0)$. Immediately after growth, the samples were annealed in vacuum at different temperatures for 15 min, and subsequently quenched to room temperature to preserve the high temperature morphology. Quenching was achieved by turning off the heater and then making the sample holder come in contact with a small liquid nitrogen vessel. For samples grown under nominally identical conditions, differences in the surface morphology were due to the different annealing conditions. The surface morphology was then studied ex situ using atomic force microscopy (AFM). Magnetic hysteresis loops were acquired, also ex situ, using the magnetooptic Kerr effect (MOKE) at room temperature.

\section{RESULTS AND DISCUSSION}

An AFM image of a $6.3 \mathrm{~nm}$ thick sample annealed at $535^{\circ} \mathrm{C}$ is shown in Fig. 1. In this case, the film is oriented along the fcc[001] direction. The pits shown in the image are as deep as the film thickness to within the AFM resolution. ${ }^{7}$ We therefore believe that the pits are devoid of Co, although we cannot exclude the possibility that a monolayer or two remain. An important observation is that the sides of the different rectangular pits on the sample are aligned along the same direction. This is reasonable given that the films are epitaxial when the pits are formed. X-ray phi scans of the sample and substrate show that the sides of the pits correspond to the Co fcc[100] and [010] directions.

Figure 2 shows the thickness dependence of the surface morphology. All of the samples shown were annealed at 


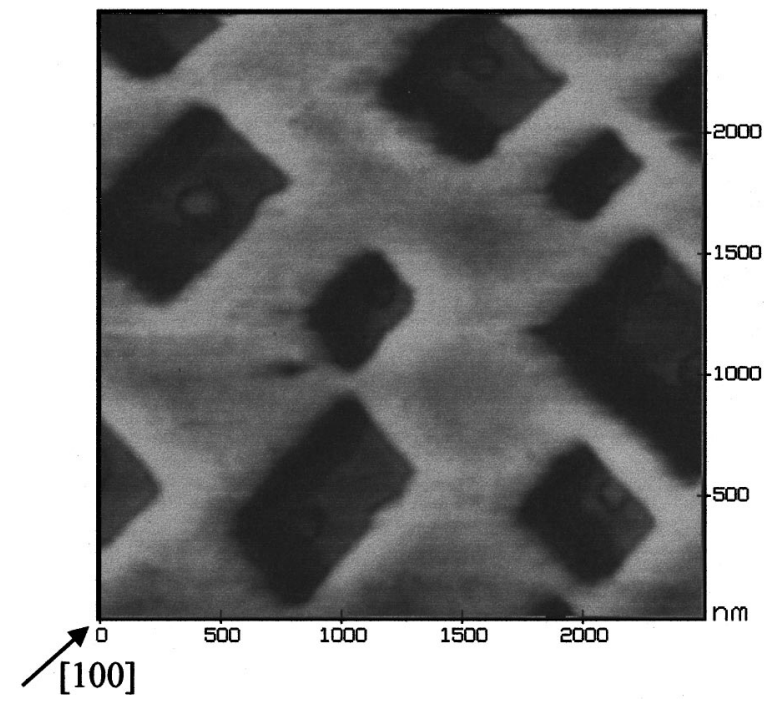

FIG. 1. Rectangular pits formed for a $6.3 \mathrm{~nm}$ thick Co film annealed at $535^{\circ} \mathrm{C}$. The film orientation is fcc[001]. The pits are aligned along the in-plane fcc[100] direction, as determined from $\mathrm{x}$-ray diffraction measurements.

$550^{\circ} \mathrm{C}$. Rectangular pits form on samples with $t<20 \mathrm{~nm}$. For the thicker sample shown, the pits are hexagonal. X-ray diffraction revealed that thicker samples remain fcc(111) oriented when they undergo their smoothening transition. Since the film is epitaxial, the pits reflect the in-plane crystal symmetry, which in this case is hexagonal.

The number of pits per unit area, $\rho$, calculated from AFM images, is shown in Fig. 3(a). For the thinner samples, $\rho$ decreases exponentially with increasing sample thickness. By fitting $\rho$ to $\rho=\rho_{0} e^{-t / \lambda}$, where $t$ is the film thickness, one obtains a characteristic length scale $\lambda=1.2 \mathrm{~nm}$. The physical meaning of $\lambda$ may correspond to the minimum film thickness required for the pits to form, but more experiments are required to verify this. Figure 3(b) shows the porosity of the film as a function of film thickness, calculated as the percentage of the area that is missing from the film. For small thickness, the porosity seems to level off, indicating that the size of the pits decreases slightly for very thin films. The exponential dependence is an indication that the pit-forming mechanism is strongly influenced by the surface and/or the substrate/film interface.

The magnetic properties of the thin fcc(001) samples which have undergone the smoothening transition are very different from those of the as-grown samples. The as-grown samples do not display an in-plane magnetic anisotropy. On the other hand, the magnetic hysteresis loops of the fcc(001) samples are strongly dependent on the direction of the applied field, as shown in Fig. 4. The presence of the double loops for angles at approximately $90^{\circ}$ from the easy axis is an indication of biaxial anisotropy, with the magnitude of the magnetic anisotropy being greater for one of the easy axes $\left(\Theta=0^{\circ}\right)$ than the other $\left(\Theta=90^{\circ}\right) .{ }^{9}$ From in-plane x-ray phi scans, ${ }^{7}$ we find that the axis with the larger anisotropy points along the fcc[110] direction, which is parallel to the in-plane $c$ axis of the $\mathrm{Al}_{2} \mathrm{O}_{3}$ substrate. A similar phenomenon has been previously observed in $\mathrm{Fe}$ bcc(001) films grown on $\mathrm{MgO}$, resulting from the angle of the Fe flux during deposi-

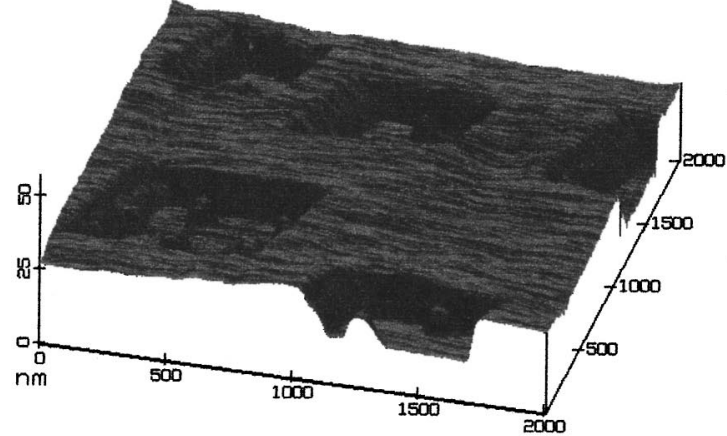

$t=8.6 \mathrm{~nm}$

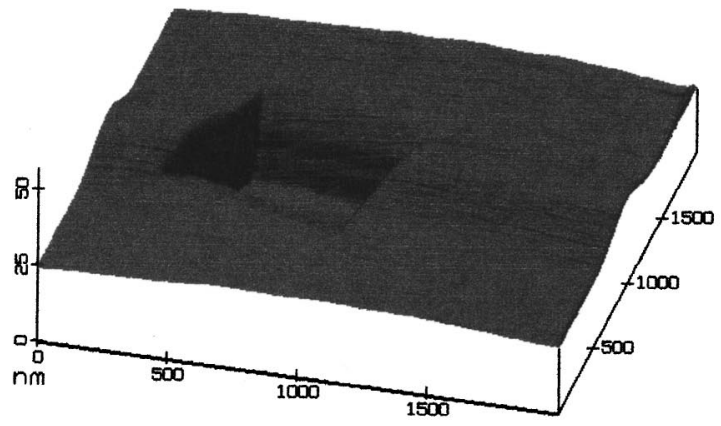

$t=18 \mathrm{~nm}$

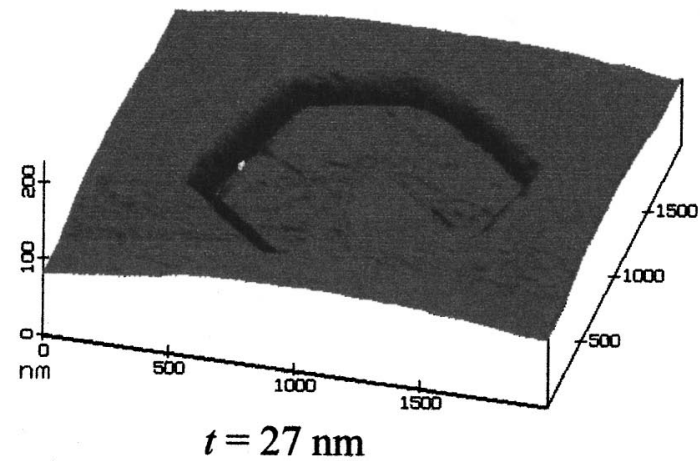

FIG. 2. AFM images of films annealed at $550{ }^{\circ} \mathrm{C}$ with different thickness $t$. Thinner films form rectangular pits, while thicker films form hexagonal pits. The $x-y$ scale is $2000 \mathrm{~nm} \times 2000 \mathrm{~nm}$. The depth of the pits corresponds to the film thickness.

tion which presumably creates a unidirectional strain. ${ }^{9}$ In our case the in-plane strain, if present, is not created during growth, but during the post-growth annealing process.

Regarding the possible mechanism for the reentrant smoothening transition and the crystallographic changes associated with it, we note that the pits are formed only on samples grown in our high vacuum sputtering system. We have performed preliminary experiments on samples grown via molecular beam epitaxy (base pressure $7 \times 10^{-10}$ Torr), and discovered that these samples do not form rectangular pits, although hexagonal pits are sometimes observed-that is, there is no $\mathrm{fcc}(111)$ to $\mathrm{fcc}(001)$ recrystallization. This points to a surface contaminant being responsible for the recrystallization, present either during growth or immediately after growth. When the contaminant desorbs at higher temperatures (approximately $550^{\circ} \mathrm{C}$ ), the surface relaxes, re- 


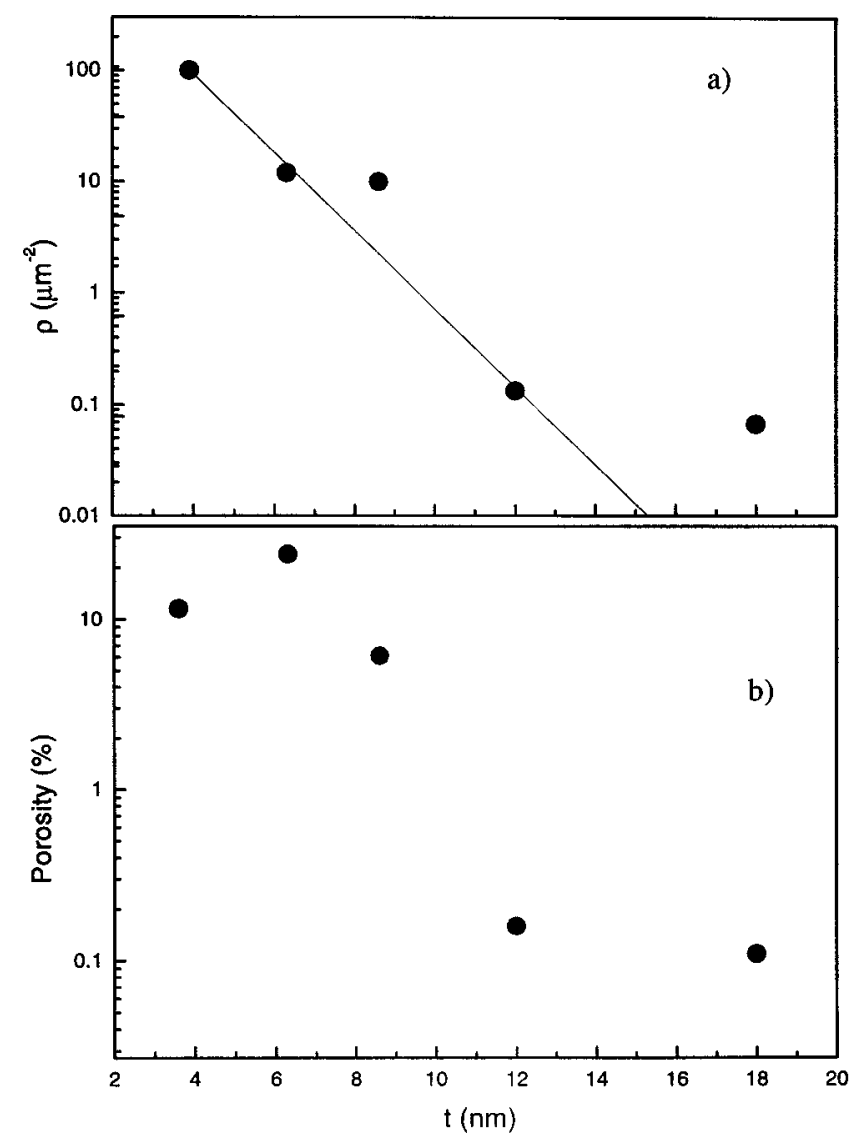

FIG. 3. Pit density (a) and porosity (b), or the percentage of the AFM image left uncovered by the film, as functions of film thickness. The solid line is a fit to $\rho=\rho_{0} e^{-t / \lambda}$ for $t<14 \mathrm{~nm}$, with $\rho_{0}=2.3 \times 10^{3} \mu \mathrm{m}^{2}$ and $\lambda=1.2 \mathrm{~mm}$.

crystallizing the film if it is thin enough. The surface contaminant therefore acts as a surfactant during the smoothening process. This smoothening mechanism has been in $\mathrm{Pt}$ surfaces contaminated with oxygen, which acts as a surfactant and thus helps create very smooth homoepitaxial Pt thin films. ${ }^{10}$ This possibility is currently being studied further.

\section{CONCLUSIONS}

We have studied the morphological and magnetic properties of $\mathrm{Co}$ thin films grown on $\mathrm{Al}_{2} \mathrm{O}_{3}$ annealed at higher temperatures. The films display a remarkable surface smoothening transition between 500 and $600^{\circ} \mathrm{C}$. For films thinner than $20 \mathrm{~nm}$, the smoothening transition coincides with a recrystallization of the entire film from fcc(111) to fcc(001) and the formation of submicron rectangular pits. For thicker films, the surface can also smoothen out, and the shape of the pits that do appear is hexagonal because their structure remains fcc(111). The pit concentration decreases exponentially with the film thickness, indicating that this is a surface effect. The magnetic properties of the fcc(001) samples are highly anisotropic, consistent with an in-plane biaxial anisotropy with two unequal easy axes. A possible mechanism for the formation of the rectangular pits is the adsorption of contaminants during or immediately after growth, followed by desorption at higher temperatures. If a method is developed for controlling the size and position of

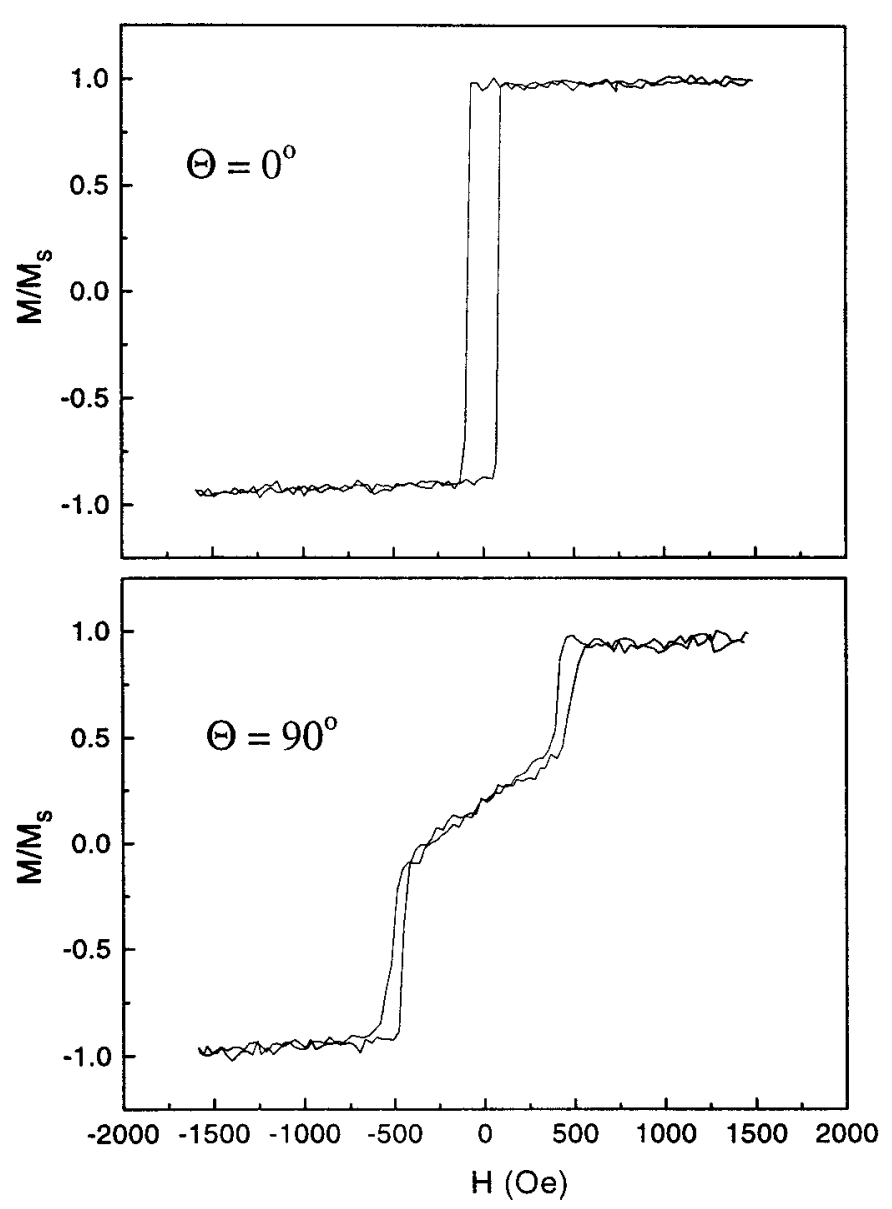

FIG. 4. Magneto-optic Kerr effect (MOKE) at room temperature in air from a fcc(001)-oriented sample which was annealed at $550{ }^{\circ} \mathrm{C}$ which contains rectangular pits. $\Theta$ is the angle between the applied magnetic field and the easy axis in the sample plane, fcc[110].

the pits, the pits could be used in liftoff nanolithography for quantum electronics and in developing new submicron magnetic structures.

\section{ACKNOWLEDGMENTS}

This work was supported by the National Science Foundation (CAREER Award No. DMR-9734051) and the American Chemical Society's Petroleum Research Fund (Starter Grant No. 32814-G5).

${ }^{1}$ Constitution of Binary Alloys, edited by M. Hansen (McGraw-Hill, New York, 1958), p. 471ff.

${ }^{2}$ Ch. Morawe, A. Stierle, N. Metoki, K. Brohl and H. Zabel, J. Magn. Magn. Mater. 102, 223 (1991).

${ }^{3}$ Y. Fukano, J. Appl. Phys. 13, 1001 (1974); C. G. Granqvist and R. A. Buhrman, ibid. 47, 2200 (1976) J. P. Chen, C. M. Sorensen, K. J. Klabunde, and G. C. Hadjipanayis, ibid. 76, 6316 (1994); J. Jiao, S. Seraphin, X. Wang, and J. C. Withers, ibid. 80, 103 (1996).

${ }^{4}$ J. M. Gallego, S. Kim, T. J. Moran, D. Lederman, and I. K. Schuller, Phys. Rev. B 51, 2550 (1995).

${ }^{5}$ G. A. Prinz, Phys. Rev. Lett. 54, 1051 (1985).

${ }^{6}$ N. Sato, J. Appl. Phys. 67, 4462 (1990) R. Krishnan, H. O. Gupta, H. Lassri, C. Sella, and M. Kaabouchi, ibid. 70, 6471 (1991).

${ }^{7}$ H. Shi and D. Lederman, Phys. Rev. B 58, R1778 (1998).

${ }^{8}$ A. R. Smith, K.-J. Chao, Q. Niu, and C.-K. Shih, Science 273, 226 (1996).

${ }^{9}$ O. Durand, J. R. Childress, P. Galtier, R. Bisaro, and A. Schuhl, J. Magn. Magn. Mater. 145, 111 (1995).

${ }^{10}$ S. Esch, M. Hohage, T. Michely, and G. Comsa, Phys. Rev. Lett. 72, 518 (1994) 